\title{
Retinogeniculate Axons Undergo Eye-Specific Segregation in the Absence of Eye-Specific Layers
}

\author{
Gianna Muir-Robinson, ${ }^{1}$ Bryan J. Hwang, ${ }^{2}$ and Marla B. Feller ${ }^{1}$ \\ ${ }^{1}$ Neurobiology Section, Biology Division, University of California San Diego, La Jolla, California 92093, and ${ }^{2}$ Howard \\ Hughes Medical Institute-National Institutes of Health Research Scholars Program, Bethesda, Maryland 20892
}

\begin{abstract}
Spontaneous retinal activity mediated by cholinergic transmission regulates the segregation of retinal ganglion cell axons in the lateral geniculate nucleus of the thalamus into eye-specific layers. The details of how the layers form are unknown. Mice lacking the $\beta 2$ subunit of the neuronal nicotinic acetylcholine receptor lack ACh-mediated waves and as a result, do not form eye-specific layers at any stage of development. However, during the second postnatal week, $\beta 2-/-$ mice have glutamate-mediated waves. Here we show that after the first postnatal week, even in the
\end{abstract}

The development of precise neural circuitry involves activitydependent refinement of an initially crude set of connections. This process has been studied extensively in the visual system of binocular animals, where retinal ganglion cell axons from the two eyes organize into eye-specific layers in the lateral geniculate nucleus (LGN). These layers are examples of macroscopic structures reflecting bulk organization of thousands of axons on the millimeter-length scale in ferrets and cats and the hundreds of micron-length scale in mice. The proposed mechanisms underlying the development and plasticity of these structures are based on activity-dependent segregation of axons representing the two eyes, a process that occurs on a much smaller length scale involving the formation, elimination, and reorganization of individual synaptic connections (Katz and Shatz, 1996; Cline, 1998; Yuste and Sur, 1999). However, the mechanism by which these synaptic rearrangements can lead to formation of macroscopic structures has not been studied directly.

Retinal ganglion cell axons segregate from an initially intermingled projection into distinct eye-specific layers in the LGN, a process that is complete in mice by postnatal day 8 (P8) (Godement et al., 1984; So et al., 1990; Upton et al., 1999; Pham et al., 2001). This segregation is driven by spontaneous retinal activity (Penn et al., 1998), called retinal waves (Meister et al., 1991; Wong et al., 1993), which is mediated by cholinergic synaptic transmission in the first postnatal week but by glutamatergic transmission in the second postnatal week (Bansal et al., 2000;

Received Feb. 26, 2002; revised April 10, 2002; accepted April 19, 2002.

This work was supported by Klingenstein Foundation and Whitehall Foundation grants (G.M.-R., M.B.F.) and by the Howard Hughes Medical Institute-National Institutes of Health Research Scholars Program (B.J.H.). We thank J. El-Maasri for technical support.

Correspondence should be addressed to Marla B. Feller, Neurobiology Section 0357, University of California San Diego, 9500 Gilman Drive, La Jolla, CA 920930357. E-mail: mfeller@ucsd.edu.

Copyright (C) 2002 Society for Neuroscience $0270-6474 / 02 / 225259-06 \$ 15.00 / 0$ absence of layers, retinothalamic axons segregate into an unlayered, patchy distribution of eye-specific regions. These results indicate that spontaneous neural activity may independently regulate eye-specific segregation and the formation of layers at the developing retinothalamic projection.

Key words: retinogeniculate segregation; retinal waves; spontaneous activity; visual-system development; eye-specific layers; nicotinic receptor subunits
Wong et al., 2000; Zhou and Zhao, 2000; Sernagor et al., 2001).

Transgenic mice lacking the $\beta 2$ subunit of the neuronal nicotinic acetylcholine receptor (nAChR) (Xu et al., 1999) lack AChmediated waves between P1 and P7 (Bansal et al., 2000). Consistent with the requirements for retinal activity in driving the formation of eye-specific layers, $\beta 2-/-$ mice fail to form normal eye-specific layers (Hwang et al., 2000; Rossi et al., 2001). Based on the assumption that poor macroscopic patterning reflects a failure of an activity-dependent segregation process, it has been argued that retinogeniculate axons remain wholly unsegregated in $\beta 2-/-$ mice (Rossi et al., 2001).

Here we demonstrate that retinogeniculate axons can segregate independently of the formation of eye-specific layers. Doublelabeling of retinogeniculate terminals from both eyes reveals that although $\beta 2-/-$ mice do not form eye-specific layers at any stage of development, retinogeniculate projections still segregate into a patchy distribution. These findings demonstrate that segregation of left eye and right eye axons can be uncoupled from macroscopic patterning in the visual system.

\section{MATERIALS AND METHODS}

Visualization of retinogeniculate projections. All surgeries on mouse pups were performed according to institutional guidelines and approved protocols. Animals were anesthetized with $3.5 \%$ isoflurane $/ 2 \% \mathrm{O}_{2}$. The eyelid was cut open to expose the temporal portion of the eye, and $0.1-1$ $\mu \mathrm{l}$ of $2 \%$ cholera toxin in $0.2 \%$ DMSO conjugated to either fluorescein isothiocyanate (FITC) or tetramethylrhodamine isothiocyanate (TRITC) (List Biological, Campbell, CA) was injected into the retina with a fine glass micropipette with a picospritzer (World Precision Instruments, Sarasota, FL). Cholera toxin is transported throughout retinal ganglion cells, clearly labeling axons and terminals. Mice were analyzed after $24 \mathrm{hr}$ (at P8, P14, and P28). Animals were heavily anesthetized with an overdose of isoflurane. After fixation by cardiac perfusion with $4 \%$ paraformaldehyde in PBS, brains were sectioned coronally to $100 \mu \mathrm{m}$ on a Vibratome.

Image analysis. Eight-bit tagged image file format images were acquired for TRITC- and FITC-labeled sections of the LGN with a CCD camera (Optronics, Goleta, CA) attached to a Zeiss (Thornwood, NY) Axioscope 2 with a $10 \times$ objective (numerical aperture, 0.45). The 


\begin{tabular}{|c|c|c|c|c|}
\hline Age & $\%$ Ipsilateral $^{a}$ & dLGN area ${ }^{b}\left(\mathrm{~mm}^{2}\right)$ & $\begin{array}{l}\text { dLGN length along the } \\
\text { dorsoventral axis }{ }^{c}(\mu \mathrm{m})\end{array}$ & $\begin{array}{l}\text { Location of ipsilateral } \\
\text { geometric center }^{d}(\mu \mathrm{m})\end{array}$ \\
\hline \multicolumn{5}{|l|}{ P8 } \\
\hline $\mathrm{WT}(n=7)$ & $16.0 \pm 1.9$ & $0.150 \pm 0.01$ & $637.6 \pm 39.2$ & $68.0 \pm 27.9$ \\
\hline$\beta 2-/-(n=9)$ & $23.9 \pm 7.8^{*}$ & $0.134 \pm 0.02$ & $552.5 \pm 54.4$ & $108.6 \pm 24.0$ \\
\hline \multicolumn{5}{|l|}{$\mathrm{P} 14$} \\
\hline $\mathrm{WT}(n=6)$ & $12.0 \pm 2.4$ & $0.245 \pm 0.02$ & $811.7 \pm 52.9$ & $93.4 \pm 13.0$ \\
\hline$\beta 2-/-(n=6)$ & $18.5 \pm 5.9^{*}$ & $0.203 \pm 0.02$ & $707.0 \pm 59.4^{*}$ & $162.3 \pm 17.9^{*}$ \\
\hline \multicolumn{5}{|l|}{ P28 } \\
\hline $\mathrm{WT}(n=4)$ & $16.1 \pm 2.0$ & $0.262 \pm 0.02$ & $839.3 \pm 49.4$ & $69.9 \pm 43.1$ \\
\hline$\beta 2-/-(n=4)$ & $28.1 \pm 5.7^{*}$ & $0.266 \pm 0.04$ & $821.8 \pm 27.9$ & $135.7 \pm 14.4^{*}$ \\
\hline
\end{tabular}

Mean values for the middle third of the dLGN ( \pm SD) are shown.

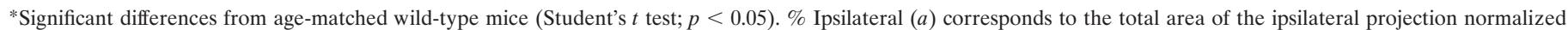

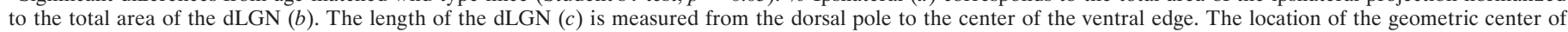

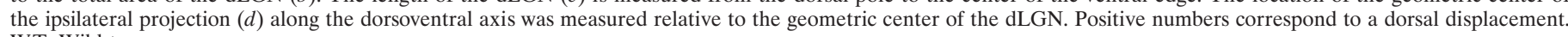
WT, Wild type.

TRITC and FITC images were digitized independently. Only the three sections that contained the largest ipsilateral projection (corresponding the middle third of the LGN) were analyzed. Background fluorescence was subtracted from sections using a rolling ball filter (NIH Image) and the gray scale was renormalized so that the range of gray-scale values was from 0 to 256 . Two kinds of image analysis were then performed on these images. Because we found that the FITC-labeled ipsilateral/TRITClabeled contralateral projections had a consistently better signal-to-noise ratio, we conducted our comparisons on these projections. We found similar results when we used the TRITC-labeled ipsilateral/FITClabeled contralateral projections.

First, to determine the parameters presented in Table 1 [i.e., the size of the dorsal LGN (dLGN) and the spatial extent and location of the ipsilateral projection], images were binarized (Metamorph software; Universal Imaging, West Chester, PA) The boundaries of the LGN were defined as the perimeter that excluded label in the extrageniculate optic tract fibers, the ventral LGN, and the intrageniculate leaf. The thresholds for both contralateral and ipsilateral axons were chosen at the location of the gray-scale histogram of the images at which there was a clear delineation between the remaining background and signal fluorescence. In general, this corresponded to a threshold value of 30-50 on the range of $0-256$. Statistical analyses of measurements comparing wild-type and $\beta 2-/-$ parameters at a given threshold were performed using a Student's $t$ test. In addition, we used these binary images in P28 wild-type mice and $\beta 2-/-$ mice to determine the sizes of the patches of ipsilateral projections. These "islands" were defined as the noncontiguous projections distinct from the major contiguous projection in the dorsal portion of the LGN that were $>10 \mu \mathrm{m}^{2}$. Projections that were $<10 \mu \mathrm{m}^{2}$ could not be measured reliably.

Second, the same background-subtracted and renormalized images were analyzed for segregation of ipsilateral and contralateral axons using Igor Software (WaveMetrics, Lake Oswego, OR). Only the regions around the ipsilateral projection were analyzed. These methods are described in Results. The image analysis was done blind to genotype of the mice.

The methods used for fluorescence imaging and electrophysiology have been described previously (Bansal et al., 2000).

\section{RESULTS}

\section{$\beta 2-/-$ mice fail to form eye-specific layers at any stage of development}

Mice lacking the $\beta 2$ subunit of the neuronal nAChR (Xu et al., 1999) lack ACh-mediated waves between P1 and P7 but have glutamate-mediated waves from P8 to P13 (Bansal et al., 2000). We used intraocular injections of two different fluorescently labeled $\beta$-cholera toxins to visualize axon terminal fields from both eyes simultaneously in coronal sections of the LGN (Fig. 1). We compared retinogeniculate projections in wild-type and $\beta 2-/-$ mice by characterizing the projections at P8, P14, and P28. In all wild-type cases, eye-specific regions were well defined and highly stereotyped, consistent with normal development of layers by P8. That is, contralateral axons occupied the majority of LGN territory but were strictly excluded from the dorsomedial region where the ipsilateral cluster terminated (Fig. 1, two left columns). In contrast, in $\beta 2-/-$ mice, eye-specific projection patterns were always poorly formed (Fig. 1, two right columns). Contralateral axons were present throughout the entire LGN and were not excluded from the region normally occupied by ipsilateral axons. Ipsilateral projections were more diffuse than normal and arranged in an irregular pattern throughout the dorsomedial and dorsolateral LGN. Ipsilateral axons were highly concentrated at the dorsal pole at all ages but extended into ventromedial regions between P14 and P28 that were normally occupied exclusively by contralateral axons. These data are summarized in Table 1. In addition, ipsilateral axons cluster into noncontiguous islands. The size of these islands was measured from fluorescence images of P28 $\beta 2-/-$ and wild-type mice. In P28 $\beta 2-/-$ mice, there were $164 \pm 13$ islands ranging in size from 10 to $500 \mu \mathrm{m}^{2}$ with a median size of $25 \pm 2 \mu \mathrm{m}^{2}(n=4)$. In contrast, in P28 wild-type mice, there were $55 \pm 5$ islands ranging in size from 10 to 300 $\mu \mathrm{m}^{2}$ with a median size of $20 \pm 2 \mu \mathrm{m}^{2}(n=4)$.

Importantly, normal layers fail to emerge in P14 or P28 $\beta 2-/-$ mice despite the presence of normal glutamate-mediated waves in these mice from P8 to P14 and despite visual experience from P14 to P28 (Rossi et al., 2001). $\beta 2+/-$ mice were indistinguishable from wild type both in retinal wave properties (Bansal et al., 2000) and in retinogeniculate patterning into eye-specific layers $(n=6$; data not shown). Comparison between $\beta 2-/-$ and wild-type Nissl-stained and 4',6'-diamidino-2-phenylindolelabeled LGN sections showed no qualitative difference in LGN cell density or size (data not shown). These findings indicate that nAChR signaling either in the retina (Feller et al., 1996; Penn et al., 1998) or in the LGN (Cimino et al., 1995; Lena and Changeux, 1999) is required for segregation of retinogeniculate axons into eye-specific layers (Rossi et al., 2001).

\section{Retinogeniculate axons from the two eyes segregate into an unlayered pattern in $\beta 2-/-$ mice}

In previous experiments, layer formation was interpreted to reflect the success or failure of eye-specific segregation. Thus, in experiments in which pharmacological (Penn et al., 1998) and genetic manipulations (Upton et al., 1999; Rossi et al., 2001) prevented the formation of normal eye-specific layers, it was 


\section{Wildtype}

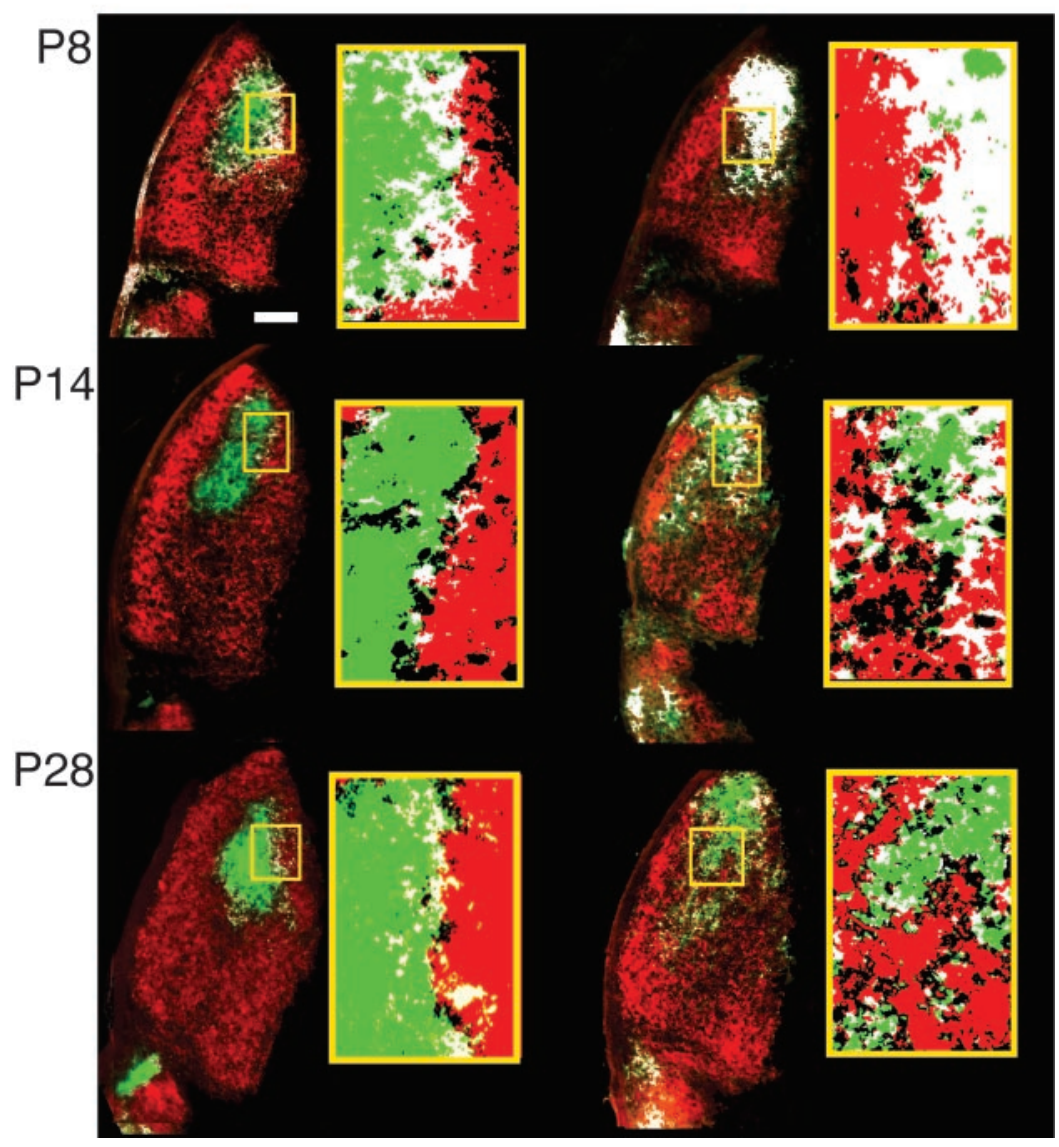

$\beta 2-1-$
Figure 1. Retinogeniculate projections are not organized into eye-specific layers in $\beta 2-/-$ mice. A pseudocolor representation of retinogeniculate projections in wild-type and $\beta 2-/-$ mice is shown. Projections from the ipsilateral projecting eye were labeled with FITC-conjugated choleratoxin (green), whereas the projections from the contralateral eye were labeled with a TRITC-conjugated construct (red). White corresponds to pixels for which there is overlap between ipsilateral and contralateral axons. At all ages, wildtype mice (two left columns) show clear segregation of ipsilateral and contralateral axons. $\beta 2-/-$ mice (two right columns) have a diffuse ipsilateral projection and no clear hole in the contralateral projection, indicating a lack of complete segregation. Boxed regions are shown at higher magnification to emphasize complex segregation patterns. Scale bar, $100 \mu \mathrm{m}$. concluded that retinogeniculate axons remain wholly unsegregated. By taking advantage of our two-color method of labeling retinal projections from the two eyes, we found, surprisingly, that despite the lack of eye-specific layers in $\beta 2-/-$ mice, there was development of a patchy eye-specific segregation of ipsilateral and contralateral fluorescent label. Terminals in $\beta 2-/-$ mice were largely unsegregated at P8, but axons from the two eyes became segregated by P14, despite the persistence of the unlayered pattern. Between P14 and P28, segregation increased and became comparable with that seen in wild-type animals that had well defined eye-specific layers (Fig. 1, two right columns). Based on these findings, we hypothesize that spontaneous activity during the second postnatal week and sensory activity in the third and fourth postnatal weeks may drive the segregation of right and left eye axons but is not sufficient to induce the formation of eyespecific layers.

To quantify the development of segregation in $\beta 2-/-$ mice, we have computed the amount of colocalization of ipsilateral and contralateral axons. This method is independent of the threshold delineating signal from background for the contralateral projection. This is especially important when examining the contralateral projection, because there are a significant number of axons that traverse the binocular region of the LGN, making the fluorescence level in the nonterminal-containing regions significantly above background. Hence, changing the threshold can significantly change the details of the projection patterns, such as the extent of overlap of ipsilateral and contralateral projecting axons in the binocular region of the LGN (Fig. 1).
Figure $2 A$ contains examples of scatter plots in which each point represents the fluorescence intensity for each $1 \mu \mathrm{m}^{2}$ pixel of the contralateral projection (Fig. $2 A$, left column) versus the fluorescence intensity of the ipsilateral projection in the same pixel (Fig. 2A, middle column). If ipsilateral and contralateral axons are well segregated, the individual points are inversely related (i.e., there is high contralateral intensity where there is low ipsilateral intensity) (e.g., P8 wild type). In contrast, if there is a substantial amount of overlap, the individual points have a positive correlation (i.e., contralateral and ipsilateral intensities will be high or low together) (e.g., P8 $\beta 2-/-$ ). From these examples, it is apparent that eye-specific segregation is initially absent in $\beta 2-/-$ mice (P8) but emerges with subsequent development (P28).

A summary of the results for all P8, P14, and P28 wild-type and $\beta 2-/-$ mice is shown in Figure $2 B$. These "segregation plots" are generated by computing the fraction of fluorescent pixels of the ipsilateral projection that contain fluorescence signal less than a critical threshold in the corresponding pixels of the contralateral projection. The curves correspond to the value of this fraction as a function of all possible threshold values of the contralateral image. A contralateral threshold of 0 implies that all pixels in the contralateral image are considered "signal;" hence all ipsilateral axons are colocalized with contralateral axons and the resulting segregation fraction $=0$. If the contralateral threshold is set at the maximum pixel value, no pixels in the contralateral image are signal; hence all ipsilateral axons are segregated and the resulting segregation fraction $=1$. The extent of segregation in two different LGNs can be compared by directly comparing these fractions 


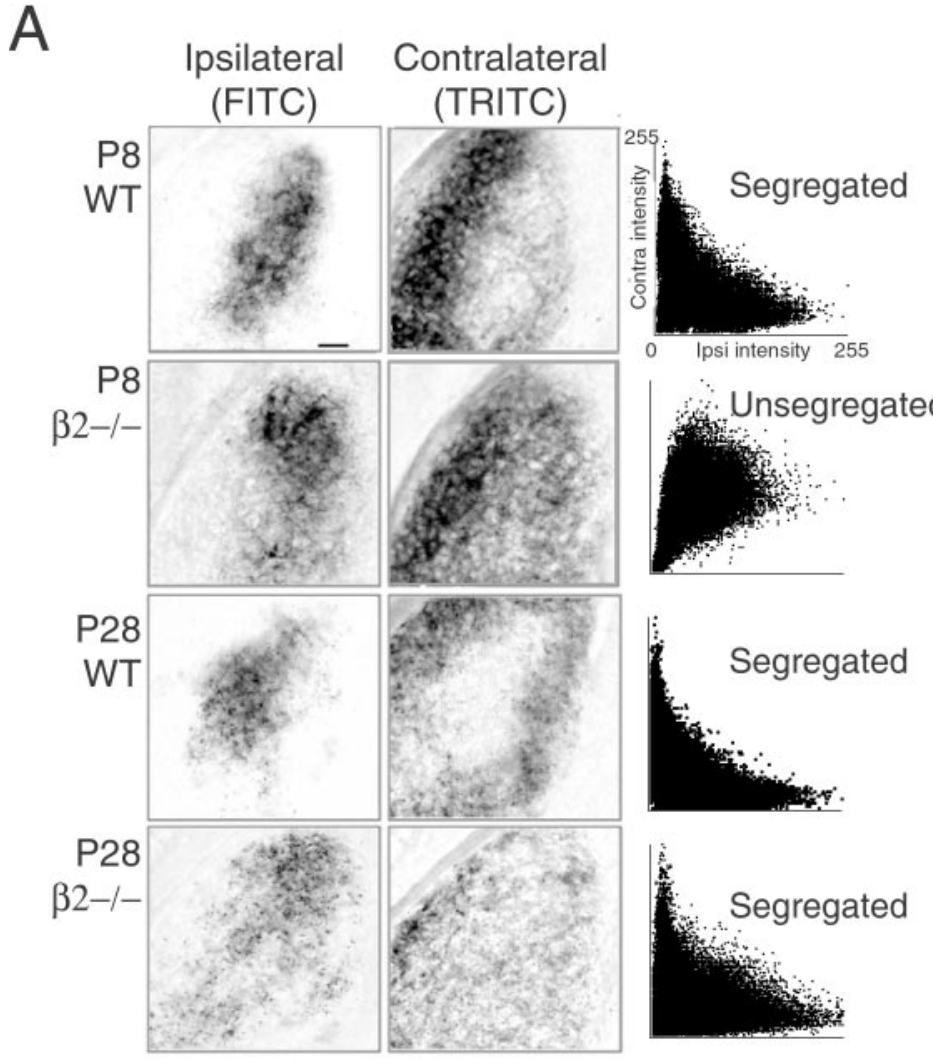

C

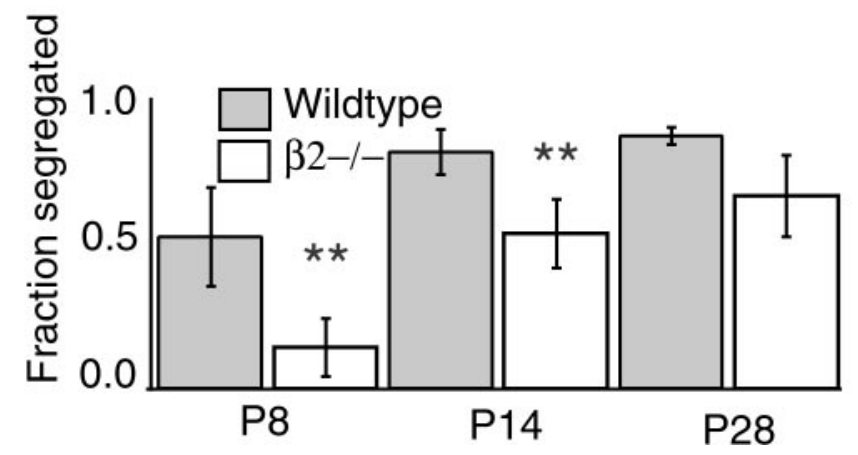

B
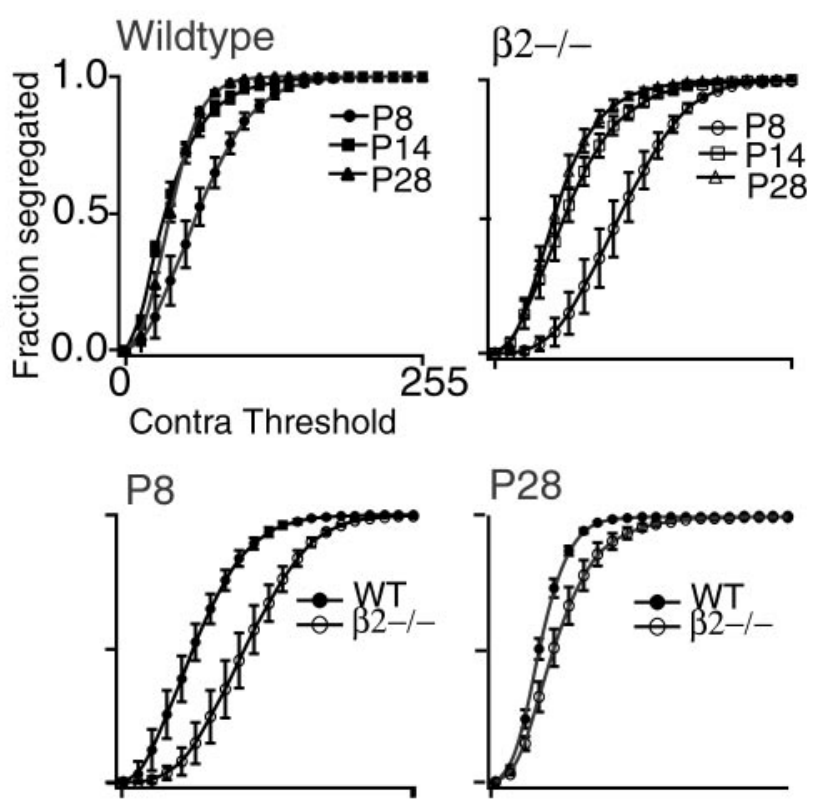

Figure 2. Segregation of ipsilateral and contralateral retinogeniculate projections. A, Fluorescence image of an ipsilateral (left) and contralateral (middle) projection in the same LGN section. Right, Scatter plot of contralateral and ipsilateral intensity for each pixel in images. Pixel resolution, $1 \mu \mathrm{m}$. Scale bar, $50 \mu \mathrm{m}$. $W T$, Wild type. $B$, Segregation plots compare the extent of segregation independent of contralateral thresholds. Traces that have lower segregation fractions represent retinogeniculate projections that are less segregated than traces that have higher values. Each trace is the average of four brains ( 3 sections per brain); error bars represent SD. Identical results were obtained for the other side of the brain [i.e., FITC labeling the contralateral projection and TRITC labeling the ipsilateral projection]. $C$, Comparison of the fraction of pixels representing segregated ipsilateral fibers at a single contralateral threshold. This is the threshold that was chosen to generate the pseudocolor images of Figure 1. At P8 and at P14, projections in $\beta 2-1-$ mice are significantly less segregated than those in wild-type mice $\left({ }^{*} p<0.01\right)$. By P28, the amount of segregation in $\beta 2-/-$ mice is comparable with the amount seen in wild-type mice $(p>0.05)$.

at each value of the contralateral threshold. For example, P8 wild-type mice have a lower segregation fraction than P14 wildtype mice at all values of the contralateral threshold; therefore we conclude that there is less segregation at P8 than at P14. Segregation values at a threshold of 50 are compared for demonstration (Fig. 2C). This threshold was used to generate the pseudocolor images of Figure 1.

This segregation analysis reveals novel features of retinogeniculate development in wild-type and $\beta 2-/-$ mice. First, although previous studies using monocular labeling techniques concluded that ipsilateral and contralateral axons in wild-type animals are fully segregated by P8, we found that they also segregate during the second postnatal week, reaching the adult level of segregation by P14 (Fig. 2B, left). These results imply that during the first postnatal week, the gross organization of the ipsilateral projection (location along the dorsoventral axis, contiguity, and size) are established and contralateral axons withdraw from the area occupied by ipsilateral axons. During the second week, retinogeniculate projections continue to segregate until there is virtually no detectable overlap between ipsilateral and contralateral projections (Fig. 2). Second, at P8, segregation of projections in $\beta 2-/-$ mice is significantly less refined than the segregation observed in wild-type mice (Fig. $2 C$ ), indicating that in addition to preventing gross patterning, the absence of waves in $\beta 2-/-$ mice prevented any eye-specific segregation. This observation suggests that eye-specific segregation into unlayered patterns requires nAChR-mediated waves. Third, although $\beta 2-/-$ mice never form clear eye-specific layers, ipsilateral and contralateral axons do segregate (Fig. $2 B$, right), and this segregation occurs between P8 and P28. Thus, by P28, the amount of segregation in $\beta 2-/-$ mice is comparable with the amount seen in wild-type mice (Fig. $2 C$ ), although layers are absent. These results indicate that when macroscopic organization of the projection is disrupted as it is in $\beta 2-/-$ mice, waves in the second postnatal 

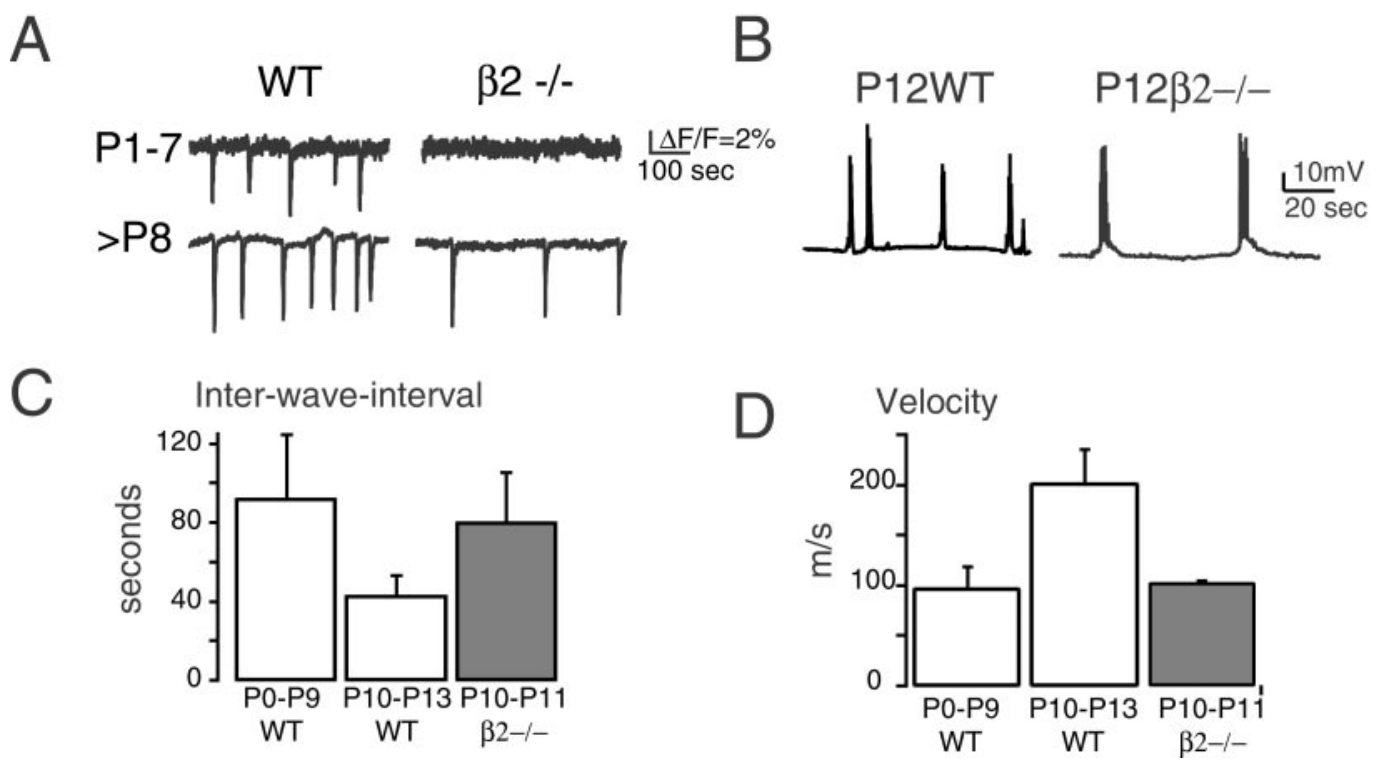

Figure 3. Comparison of the spatiotemporal properties of retinal waves in wild-type and $\beta 2-/-$ mice $A$, Fractional change in fura- 2 AM fluorescence averaged over a $200 \mu \mathrm{m}^{2}$ region of retinas from wild-type (WT) mice (left traces) and $\beta 2-/-$ mice (right traces) during the first (top) and second (bottom) postnatal weeks. Downward deflections indicate increases in intracellular calcium induced by propagating retinal waves. $B$, Traces from current-clamp recordings from presumptive ganglion cells in P12 retinas from WT (left trace) and $\beta 2-/-$ (right trace) mice. $C$, Summary data comparing the interwave interval recorded with fluorescence imaging $(n=11$ control mice between P2 and P11, $n=13$ control mice between P12 and P14, and $n=8 \beta 2-/-$ mice between P9 and P13). D, Summary data comparing the wavefront velocity ( $n=10$ waves in 3 control mice and $n=10$ waves in $3 \beta 2-/-$ mice).

week are sufficient to drive eye-specific segregation but not sufficient to induce eye-specific layer formation.

\section{Glutamate-receptor-mediated waves in $\beta 2-/-$ mice differ from those in wild-type mice}

One possible reason that waves in the second postnatal week do not induce layer formation is that correlations in retinal ganglion cell firing induced by these late waves are different from those of early waves $(<\mathrm{P} 7)$ and are not appropriate to drive macroscopic rearrangements of axonal arbors. To address this question, we compared the spatiotemporal properties of waves in the first and second postnatal weeks in wild-type and $\beta 2-/-$ mice.

Experiments were conducted on retinas isolated from newborn mice (P0-P14) and incubated in the calcium indicator fura-2 AM. The spatial distribution and time course of spontaneous changes in intracellular calcium concentration in the ganglion cell layer were assessed with real-time fluorescence imaging and represented by the fractional change in fluorescence, $\Delta F / F$ (Fig. $3 A$ ). In normal mice, during the first postnatal week, periodic changes in intracellular calcium concentrations associated with membrane depolarizations are driven by cholinergic synaptic inputs (Feller et al., 1996; Zhou, 1998). At P11, retinal waves switch from an nAChR-based to a glutamate receptor-based circuitry (Bansal et al., 2000; Wong et al., 2000; Zhou and Zhao, 2000). Compared with nAChR-mediated waves recorded in wild-type mice during the first postnatal week, glutamate receptor-mediated waves recorded in wild-type mice during the second postnatal week propagate at approximately twice the velocity and depolarize individual ganglion cells at approximately twice the frequency (Fig. $3 B-D)$. The waves recorded in $\beta 2-/-$ mice during the second postnatal week are very similar to the early waves of wild-type mice in terms of frequency of calcium transients and velocity (Fig. $3 B-D)$. The reason for the immature wave dynamics in $\beta 2-1-$ mice is unknown. However, these data indicate that the inability of retinal waves to restore eye-specific layers in the second post- natal week is not likely to be attributable to differing global retinal-activity patterns.

\section{DISCUSSION}

We found that in mice lacking the $\beta 2$ subunit of the nAChR, retinogeniculate axons segregate in the absence of eye-specific layers. In contrast to a contiguous layer in wild-type mice, ipsilateral projecting axons in $\beta 2-/-$ mice are arranged as a series of small noncontiguous islands. Many previous studies of retinogeniculate development used the existence of eye-specific layers as the sole index of retinogeniculate segregation. These findings demonstrate that eye-specific segregation can be dissociated from layer formation. This dissociation has important implications for models of how neural activity may influence retinogeniculate development.

We found that although $\beta 2-/-$ mice lack wave activity only during the first postnatal week, they do not form eye-specific layers at any point during development. This suggests that the first postnatal week may constitute a critical period for eyespecific layer formation. This finding confirms previous pharmacological (Penn et al., 1998) and genetic (Rossi et al., 2001) studies that indicate that cholinergic signaling and competition between the two eyes is necessary for of eye-specific layer formation. We found that although $\beta 2-/-$ mice have glutamate receptor-mediated waves after $\mathrm{P} 8$, this activity is not sufficient to drive layer formation. This inability of endogenous activity to drive layer formation is not likely attributable to differences in the spatiotemporal pattern, because glutamate receptor-mediated waves during the second postnatal week in $\beta 2-/-$ mice are similar to nAChR-mediated waves during the first postnatal week in wild-type mice (Fig. 3). A critical period is likely determined by a functional asymmetry between ipsilateral and contralateral projections (Crair et al., 1998), perhaps because of the timing difference between the ingrowth of ipsilateral and contralateral pro- 
jections (Godement et al., 1984) or the transient expression and/or recognition of requisite molecules (Corriveau et al., 1998; Feldheim et al., 1998; Upton et al., 1999; Land and ShamallaHannah, 2001; Pham et al., 2001). Although the exact mechanism remains to be determined, it is clear that both competition between the two eyes and the level of endogenous retinal activity are critical in establishing eye-specific layers (Stellwagen and Shatz, 2002).

Second, we found that in the second through fourth postnatal weeks, projections from the two eyes become segregated within the unlayered pattern. Despite the absence of eye-specific layers in $\beta 2-/-$ mice, we found that beginning in the second postnatal week, retinal inputs from the two eyes segregate. This suggests that activity patterns distinct from nAChR-mediated retinal waves, such as those mediated by glutamate and visual experience, can drive eye-specific segregation, perhaps through a Hebbian process (Katz and Shatz, 1996; Cline, 1998; Zhang and Poo, 2001). We observe segregation on a similar length scale in wildtype mice between the ages of P8 and P14, indicating that patchy segregation is not a compensatory artifact of the transgenic animals. Moreover, similar patchy retinogeniculate segregation patterns have been described in animals that have normal spontaneous retinal activity but do not form eye-specific layers. For example, patchy, unlayered distributions of retinogeniculate inputs form in animals that have altered numbers of retinal axons crossing at the chiasm (Guillery, 1969) and in "rewired" ferrets in which retinal input is rerouted to auditory thalamus (Angelucci et al., 1997).

Thus, the retinogeniculate projection provides a robust system in which the mechanisms of activity-dependent segregation can be studied. Experiments that manipulate endogenous activity patterns at different developmental stages in both normal and $\beta 2-/-$ mice will allow us to determine the cellular basis of the processes that drive eye-specific segregation and layer formation.

\section{REFERENCES}

Angelucci A, Clasca F, Bricolo E, Cramer KS, Sur M (1997) Experimentally induced retinal projections to the ferret auditory thalamus: development of clustered eye-specific patterns in a novel target. J Neurosci 17:2040-2055.

Bansal A, Singer JH, Hwang BJ, Xu W, Beaudet A, Feller MB (2000) Mice lacking specific nAChR subunits exhibit dramatically altered spontaneous activity patterns and reveal a limited role for retinal waves in forming $\mathrm{ON} / \mathrm{OFF}$ circuits in the inner retina. $\mathrm{J}$ Neurosci 20:7672-7681.

Cimino M, Marini P, Colombo S, Andena M, Cattabeni F, Fornasari D, Clementi F (1995) Expression of neuronal acetylcholine nicotinic receptor $\alpha 4$ and $\beta 2$ subunits during postnatal development of the rat brain. J Neural Transm Gen Sect 100:77-92.

Cline HT (1998) Topographic maps: developing roles of synaptic plasticity. Curr Biol 8:R836-R839.

Corriveau RA, Huh GS, Shatz CJ (1998) Regulation of class I MHC gene expression in the developing and mature CNS by neural activity. Neuron 21:505-520.

Crair MC, Gillespie DC, Stryker MP (1998) The role of visual experience in the development of columns in cat visual cortex. Science 279:566-570
Feldheim DA, Vanderhaeghen P, Hansen MJ, Frisen J, Lu Q, Barbacid M, Flanagan JG (1998) Topographic guidance labels in a sensory projection to the forebrain. Neuron 21:1303-1313.

Feller MB, Wellis DP, Stellwagen D, Werblin FS, Shatz CJ (1996) Requirement for cholinergic synaptic transmission in the propagation of spontaneous retinal waves. Science 272:1182-1187.

Godement P, Salaun J, Imbert M (1984) Prenatal and postnatal development of retinogeniculate and retinocollicular projections in the mouse. J Comp Neurol 230:552-575.

Guillery RW (1969) An abnormal retinogeniculate projection in Siamese cats. Brain Res 14:739-741.

Hwang B, Feldman DE, Feller MB (2000) Transgenic mice lacking retinal waves during the first postnatal week fail to form eye-specific layers in the dLGN. Soc Neurosci Abstr 26:316.

Katz LC, Shatz CJ (1996) Synaptic activity and the construction of cortical circuits. Science 274:1133-1138.

Land PW, Shamalla-Hannah L (2001) Transient expression of synaptic zinc during development of uncrossed retinogeniculate projections. J Comp Neurol 433:515-525.

Lena C, Changeux JP (1999) The role of $\beta 2$-subunit-containing nicotinic acetylcholine receptors in the brain explored with a mutant mouse. Ann NY Acad Sci 868:611-616.

Meister M, Wong RO, Baylor DA, Shatz CJ (1991) Synchronous bursts of action potentials in ganglion cells of the developing mammalian retina. Science 252:939-943.

Penn AA, Riquelme PA, Feller MB, Shatz CJ (1998) Competition in retinogeniculate patterning driven by spontaneous activity. Science 279:2108-2112.

Pham TA, Rubenstein JL, Silva AJ, Storm DR, Stryker MP (2001) The cre/creb pathway is transiently expressed in thalamic circuit development and contributes to refinement of retinogeniculate axons. Neuron 31:409-420.

Rossi FM, Pizzorusso T, Porciatti V, Marubio LM, Maffei L, Changeux JP (2001) Requirement of the nicotinic acetylcholine receptor $\beta 2$ subunit for the anatomical and functional development of the visual system. Proc Natl Acad Sci USA 98:6453-6458.

Sernagor E, Eglen SJ, Wong RO (2001) Development of retinal ganglion cell structure and function. Prog Retin Eye Res 20:139-174.

So KF, Campbell G, Lieberman AR (1990) Development of the mammalian retinogeniculate pathway: target finding, transient synapses, and binocular segregation. J Exp Biol 153:85-104.

Stellwagen D, Shatz CJ (2002) An instructive role for retinal waves in the development of retinogeniculate connectivity. Neuron 33:357-367.

Upton AL, Salichon N, Lebrand C, Ravary A, Blakely R, Seif I, Gaspar $P$ (1999) Excess of serotonin (5-HT) alters the segregation of ispilateral and contralateral retinal projections in monoamine oxidase A knock-out mice: possible role of 5-HT uptake in retinal ganglion cells during development. J Neurosci 19:7007-7024.

Wong RO, Meister M, Shatz CJ (1993) Transient period of correlated bursting activity during development of the mammalian retina. Neuron 11:923-938.

Wong WT, Myhr KL, Miller ED, Wong RO (2000) Developmental changes in the neurotransmitter regulation of correlated spontaneous retinal activity. J Neurosci 20:351-360.

$\mathrm{Xu}$ W, Orr-Urtreger A, Nigro F, Gelber S, Sutcliffe CB, Armstrong D, Patrick JW, Role LW, Beaudet AL, De Biasi M (1999) Multiorgan autonomic dysfunction in mice lacking the $\beta 2$ and the $\beta 4$ subunits of neuronal nicotinic acetylcholine receptors. J Neurosci 19:9298-9305.

Yuste R, Sur M (1999) Development and plasticity of the cerebral cortex: from molecules to maps. J Neurobiol 41:1-6.

Zhang LI, Poo MM (2001) Electrical activity and development of neural circuits. Nat Neurosci 4:1207-1214.

Zhou ZJ (1998) Direct participation of starburst amacrine cells in spontaneous rhythmic activities in the developing mammalian retina. J Neurosci 18:4155-4165.

Zhou ZJ, Zhao D (2000) Coordinated transitions in neurotransmitter systems for the initiation and propagation of spontaneous retinal waves. J Neurosci 20:6570-6577. 